\title{
Identification of the novel differentiation marker MS4A8B and its murine homolog MS4A8A in colonic epithelial cells lost during neoplastic transformation in human colon
}

\author{
J Michel ${ }^{4,1}$, K Schönhaarr ${ }^{1,4}$, K Schledzewski ${ }^{1}$, C Gkaniatsou ${ }^{1}$, C Sticht ${ }^{2}$, B Kellert ${ }^{1}$, F Lasitschka ${ }^{3}$, C Géraud ${ }^{1}$, S Goerdt ${ }^{1}$ and \\ A Schmieder ${ }^{*} 1$
}

The CD20-homolog Ms4a8a has recently been shown to be a marker for alternatively activated macrophages but its expression is not restricted to hematopoietic cells. Here, MS4A8A/MS4A8B expression was detected in differentiated intestinal epithelium in mouse and human, respectively. Interestingly, no MS4A8B expression was found in human colon carcinoma. Forced overexpression of MS4A8A in the murine colon carcinoma cell line CT26 led to a reduced proliferation and migration rate. In addition, MS4A8A-expressing CT26 cells displayed an increased resistance to hydrogen peroxide-induced apoptosis, which translated in an increased end weight of subcutaneous MS4A8A + CT26 tumors. Gene profiling of MS4A8A + CT26 cells revealed a significant regulation of 225 genes, most of them involved in cytoskeletal organization, apoptosis, proliferation, transcriptional regulation and metabolic processes. Thereby, the highest upregulated gene was the intestinal differentiation marker cytokeratin 20. In conclusion, we show that MS4A8A/MS4A8B is a novel differentiation marker of the intestinal epithelium that supports the maintenance of a physiological barrier function in the gut by modulating the transcriptome and by conferring an increased resistance to reactive oxygen species. The absence of MS4A8B in human colonic adenocarcinomas shown in this study might be a helpful tool to differentiate between healthy and neoplastic tissue.

Cell Death and Disease (2013) 4, e469; doi:10.1038/cddis.2012.215; published online 24 January 2013

Subject Category: Cancer

The mammalian large intestine is a dynamic organ involved in water, electrolyte and vitamin absorption, salvage of nutrients obtained by bacterial fermentation and transportation of feces. For the fulfilment of these physiological processes, the large intestine is equipped with a monolayer of highly specialized columnar epithelial cells lining approximately $2000 \mathrm{~cm}^{2}$ of colonic lumen folded in crypts. ${ }^{1}$ As the colonic mucosa is permanently exposed to damaging mechanical and chemical stress factors, the maintenance of a proper organ function requires rapid renewal and differentiation of mucosal cells. The renewal is achieved by a pool of asymmetrically dividing stem/progenitor cells located in the proliferative compartment of the crypt, which guarantees the preservation of multipotent daughter cells, while generating cells committed to differentiation along the crypt-luminal axis. These cells express cell-cycle-promoting genes and suppress phenotypic markers of mature states. ${ }^{2}$ Cells intended for differentiation, meanwhile, migrate along the crypt-lumen axis, exit the cell cycle and start expressing specific differentiation markers as villin $2,^{2}$ Krüppel-like factor $4,{ }^{3,4}$ gelsolin, ${ }^{5}$ calbinin $3,{ }^{6}$ cytokeratin $20(\mathrm{Krt20})^{7}$ or carbonic anhydrase $\mathrm{II}^{2,8}$ depending on their committed differentiation into colonocytes, goblet cells or enteroendocrine cells. In general, during differentiation, colonic epithelial cells downregulate cell cycle and DNA synthesis genes, while genes important for the cytoskeleton and lipid uptake are upregulated. ${ }^{9}$ After 3-5 days of migration, colonic epithelial cells reach the innermost luminal side of the colon, where they undergo apoptosis and are shed into the colonic lumen. ${ }^{10}$

The high rate of proliferation and differentiation processes found in the intestinal tract are orchestrated tightly by spatially and temporally regulated transcription factors such as the caudal-type homeobox transcription factor 2 (CDX2), hepatocyte nuclear factor 4 (HNF4), HNF1, GATA-binding protein

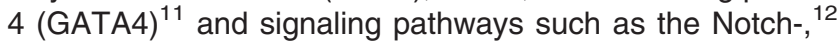
the $\mathrm{Wnt} / \beta$-catenin- ${ }^{13-15}$ and the TGF-beta signaling pathway. ${ }^{16}$ Mutations or misregulation in such central regulators ${ }^{17}$ predispose to neoplastic transformation, which leads to highly

\footnotetext{
${ }^{1}$ Department of Dermatology, Venereology and Allergology, University Medical Center and Medical Faculty Mannheim, University of Heidelberg, and Center of Excellence in Dermatoloy, Mannheim, Germany; ${ }^{2}$ Center for Medical Research, Medical Faculty Mannheim, University of Heidelberg, Mannheim, Germany and ${ }^{3}$ Institute of Pathology, University Hospital Heidelberg, Mannheim,Germany

*Corresponding author: A Schmieder, Department of Dermatology, Venereology and Allergology, University Medical Center Mannheim, University of Heidelberg, Theodor-Kutzer Ufer 1-3, Mannheim 68167, Germany. Tel: + 49621383 2048; Fax: + 49621383 3815; E-mail: astrid.schmieder@umm.de

${ }^{4}$ These authors contributed equally to this work.

Keywords: Differentiation of enterocytes; apoptosis; proliferation; keratin 20; colon carcinoma

Abbreviations: BrdU, 5-bromo-2'-deoxy-uridine; CDX2, caudal-type homeobox transcription factor 2; EV, empty vector; FACS, fluorescence-activated cell sorter; $\mathrm{H}_{2} \mathrm{O}_{2}$, hydrogen peroxide; HNF1, hepatocyte nuclear factor 1; Krt20, Cytokeratin 20; Ms4a, membrane spanning 4-domains subfamily A

Received 29.11.12; accepted 14.12.12; Edited by A Stephanou
} 
proliferative and dedifferentiated tumor cells characterized by chromosomal instability. ${ }^{18}$

In this work, we have found the CD20-homolog membrane spanning 4-domains subfamily A (MS4A)8B/MS4A8A expressed in human and murine colonic epithelial cells committed to differentiation outside the colonic crypt, while its expression is lost in human colon carcinoma.

MS4A8B and its murine homolog MS4A8A belongs to the newly defined MS4A/CD20 family of proteins characterized by four transmembrane regions. So far, the exact functions of many MS4A family members are only partially known. They seem to be involved in calcium signaling, modulation of intracellular signaling and differentiation processes of haematopoietic and epithelial cells ${ }^{19,20}$ by acting as cell-surface signaling molecules and intracellular adapter proteins. ${ }^{21}$

In this report, we show that MS4A8A, overexpressed in a colon carcinoma cell line, inhibits proliferation and migration, whereas it increases resistance against hydrogen peroxide $\left(\mathrm{H}_{2} \mathrm{O}_{2}\right)$-induced apoptosis. In addition, Ms4a8a overexpression leads to a specific gene expression profile. Especially Krt20 is upregulated, indicating its important role for the differentiation of colonic epithelial cells along the cryptluminal axis.

\section{Results}

MS4A8B/MS4A8A is expressed in human and murine intestinal epithelial cells and is lost in human colon cancer. In our previous work, the novel CD20-homolog MS4A8A was shown to be expressed by M2 macrophages in parasitic infections and by tumor-associated macrophages in murine mammary carcinoma and malignant melanoma. As a previous gene transcription analysis by Liang et al. ${ }^{22}$ revealed a strong mRNA expression of $\mathrm{Ms} 4 \mathrm{a} 8 \mathrm{a}$ in the murine colon, we used our previously published rabbit polyclonal anti mouse MS4A8A antibody to investigate the physiological expression pattern of MS4A8A in the murine intestinal tract.

Immunohistochemical stainings revealed MS4A8A to be expressed by epithelial cells of the stomach, the small and the large intestine (Figures $1 \mathrm{a}-\mathrm{c}$ ). MS4A8A was thereby not expressed in the stem cell niche of the crypts, but its expression increased along the crypt-luminal axis (Figure 1d).

To investigate whether a similar expression pattern could also be detected in the human gut, a rabbit polyclonal antibody against a specific MS4A8B peptide was generated and used to stain healthy human colon of patients suffering from colon carcinoma. The specifity of the antibody was confirmed by histochemical staining and western blot analysis of transiently transfected MS4A8B + Caco2 cells (data not shown).

Immunohistochemical analysis showed an MS4A8B expression pattern resembling the one found in mice, with MS4A8B being absent in the crypt bottom, but increasing along the mid part of the crypt-luminal axis and lacking on the innermost luminal side of the colon (Figure 1e). Interestingly, MS4A8B was either absent or detectable very faint in the colon carcinoma of these patients (Figure 1f). Western blot analysis of protein extracts from healthy colon and colon carcinomas confirmed the loss of MS4A8B in the neoplastic tissue (Figure 1g).

Overexpression of Ms4a8a in the murine colon carcinoma cell line СT26 reduces migration and proliferation, whereas resistance to apoptosis was increased. The fact that both MS4A8A and MS4A8B expression in murine and human colon increases along the crypt-surface axis and is lost during neoplastic transformation pointed to an association of MS4A8A/MS4A8B with differentiation processes in the colon. As it is known that during differentiation colonic epithelial cells downregulate genes involved in the cell cycle and upregulate genes associated with the cytoskeleton, we were interested whether MS4A8A expression exerted some effects on migration and proliferation of colonic epithelial cells. In addition, the effect of Ms4a8a on resistance to apoptosis was measured. For this purpose, two Ms4a8atransfected (CT26-\#14 and CT26-\#20) and two control empty vector (EV)-transfected (CT26-EV1 and CT26-EV2) CT26 colon carcinoma cell clones were generated. The Ms4a8a expression level of the clones was verified by immunohistochemistry (Figure 2a), fluorescence-activated cell sorter (FACS) analysis (Figure 2b), quantitative reverse transcriptase-PCR (Figure 2c) and western blot analysis (Figure 2d).

To test whether MS4A8A has an influence on cell motility, a scratch was set in the cell monolayer of confluent transgenic cells, pre-treated with mitomycin. The migration rate of the cells into the scratch was assessed by regular $2 \mathrm{~h}$ scratch measurements until complete closure. The Ms4a8a + CT26 clones \#14 and \#20 migrated significantly slower into the artificial scratch than the EV clones EV1 and EV2 (Figure 3a), with a complete closure of the scratch achieved after $10 \mathrm{~h}$ by CT26-EV1 and CT26-EV2 versus $12 \mathrm{~h}$ by CT26-\#14 and CT26-\#20.

The hypothesis that MS4A8A overexpression also interfere with cell proliferation was investigated by the 5-bromo-2'deoxy-uridine (BrdU) incorporation method. FACS analysis of CT26 transfectants revealed a statistically significant reduction in BrdU incorporation of Ms4a8a + CT26-\#14 (47\%) and CT26-\#20 (45.8\%) clones compared with CT26-EV1 (63.1\%) and CT26-EV2 (61.5\%) clones (Figure 3b). The lowered level of BrdU incorporation in Ms4a8a + CT26 cells indicates a reduced number of cell divisions and therefore a reduced proliferation rate in comparison to EV controls.

To test whether MS4A8A-mediated reduction of migration and proliferation rates coincide with a higher rate of cell mortality, we analyzed the resistance of transgenic CT26 clones to apoptosis by exposing them to $2.5-\mathrm{mm}_{2} \mathrm{O}_{2}$. Surprisingly, after $3 \mathrm{~h} 77.2 \%$ of CT26-EV1 and $65.1 \%$ of CT26-EV2 were apoptotic, while the apoptosis rate was only $39.2 \%$ in CT26-\#14 and 38.3\% in CT26-\#20 (Figure 3c). The differences in apoptosis between MS4A8A + clones versus controls were statistically significant.

Our results indicate that overexpression of MS4A8A has an inhibitory effect on proliferation and migration of transgenic CT26 cells but seems to increase resistance to apoptotic stimuli. 

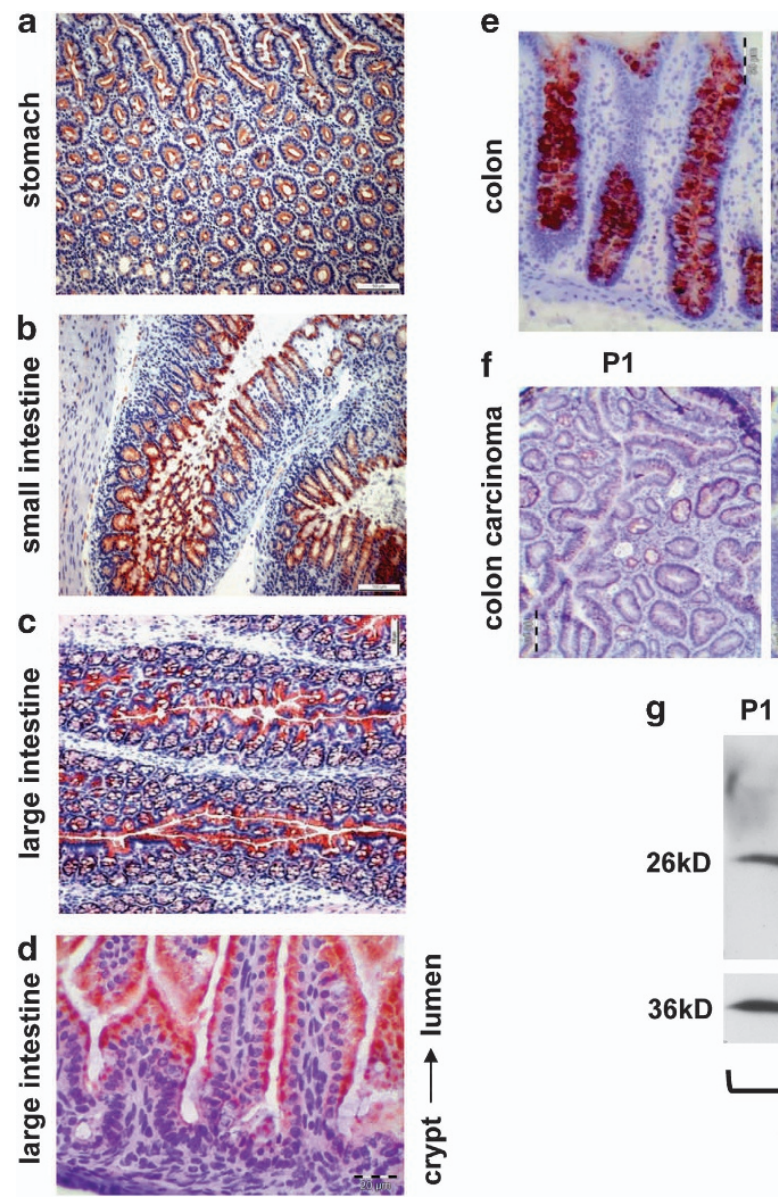

f

P1
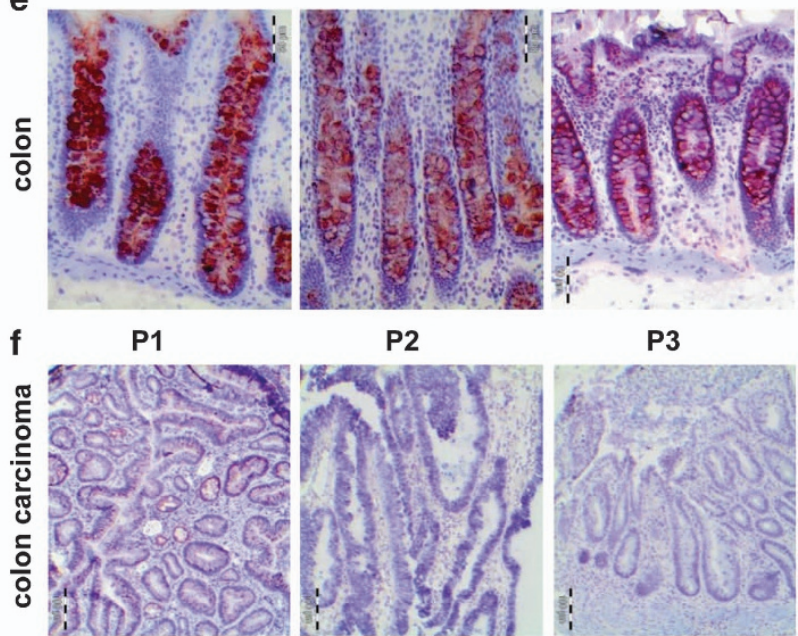

P2

P3
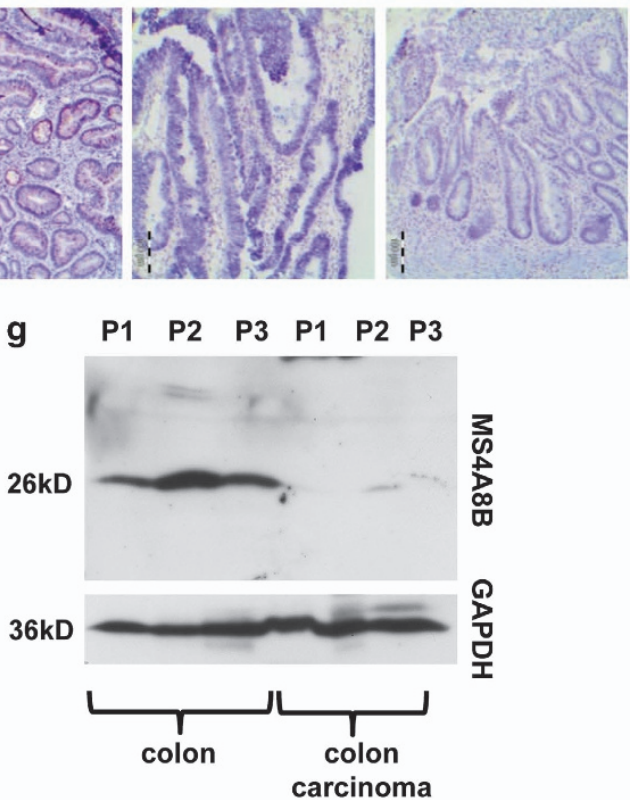

Figure 1 The homologs MS4A8A/MS4A8B are expressed in a similar manner in human and murine colonocytes, with an increase in expression along the crypt-luminal axis. The expression is restricted to normal colonic mucosa and lost in colon cancer. Histochemical stainings of acetone-fixed cryostat sections of (a) murine stomach, (b) small intestine and (c) large intestine with rabbit anti-mouse polyclonal MS4A8A antibody. Positive cells appear in red. Bars $=100 \mu \mathrm{m}$. (d) Image magnification of the crypts of murine large intestine stained with rabbit-anti mouse polyclonal MS4A8A antibody. Bar $=20 \mu \mathrm{m}$. In a-d, representative pictures of three independent stainings are shown. Histochemical stainings of acetone-fixed cryostat sections of (e) human colon and (f) colon carcinoma of three independent patients (P1-3) with rabbit anti-human polyclonal MS4A8B antibody. Positive cells appear in red. Bars $=100 \mu \mathrm{m}$ and $50 \mu \mathrm{m}$ as indicated. (g) Western blot analysis of protein extracts of tissue from healthy colon and colon carcinoma of three independent patients (P1-3) stained with rabbit anti-human polyclonal MS4A8B antibody. To ensure identical protein loading, the same samples were stained with rabbit anti-mouse/human GAPDH antibody; $n=3$

Subcutaneous injection of MS4A8A + CT26 clones in the mouse flank leads to an increased tumor end weight attributable to a higher resistance to apoptosis. To test whether the reduced migration and proliferation or the higher resistance to apoptosis assessed in MS4A8A + CT26 clones in vitro translates in a differential tumor growth in vivo, a total of $3 \times 10^{5}$ transgenic CT26-\#14 and CT-EV1 tumor cells were injected in the right mouse flank. In vivo, MS4A8A+ CT26 tumors reached a significantly higher relative tumor end weight after 14 days (Figure $4 \mathrm{a}$ ). To confirm this result independently, the experiment was repeated with CT26-\#20 and CT6-EV2. Even after a growth period of 21 days, the end weight of MS4A8A + tumors exceeded the size of controls significantly (Figure 4b).

To test whether the apoptosis resistance measured for MS4A8A + CT26 clones in vitro is still detectable in solid tumors in vivo, single-cell apoptosis rate was assessed by staining representative cryosections of CT26-\#14 and CT26EV2 tumors with a rabbit anti-mouse caspase 3 antibody.
In CT26-\#14 tumors, a mean of 1.8 caspase 3 -positive cells per $\times 40$ high power field was counted, while in CT26-EV2, the rate of caspase 3-positive cells was significantly higher with a mean of 27.1. Necrotic areas correlated with the total tumor weight and were excluded from this analysis (Figure 4c).

Therefore, a higher resistance of MS4A8A + CT26 cells to apoptosis measured in vitro and in vivo could be one relevant factor causing the increased tumor end weight.

MS4A8A + CT26 cells are characterized by an overexpression of Krt20 and other genes. A gene microarray analysis was performed to identify the molecular program underlying the effects of MS4A8A + CT26 cells on proliferation, migration and apoptosis.

The comparison of the gene expression profile of CT26-\#14 and CT26-\#20 with CT26-EV1 and CT26-EV2 showed a significant regulation of 225 genes, thereof 148 genes were upregulated and 77 genes were downregulated (Table 1). Several well-known genes involved in cytoskeletal 

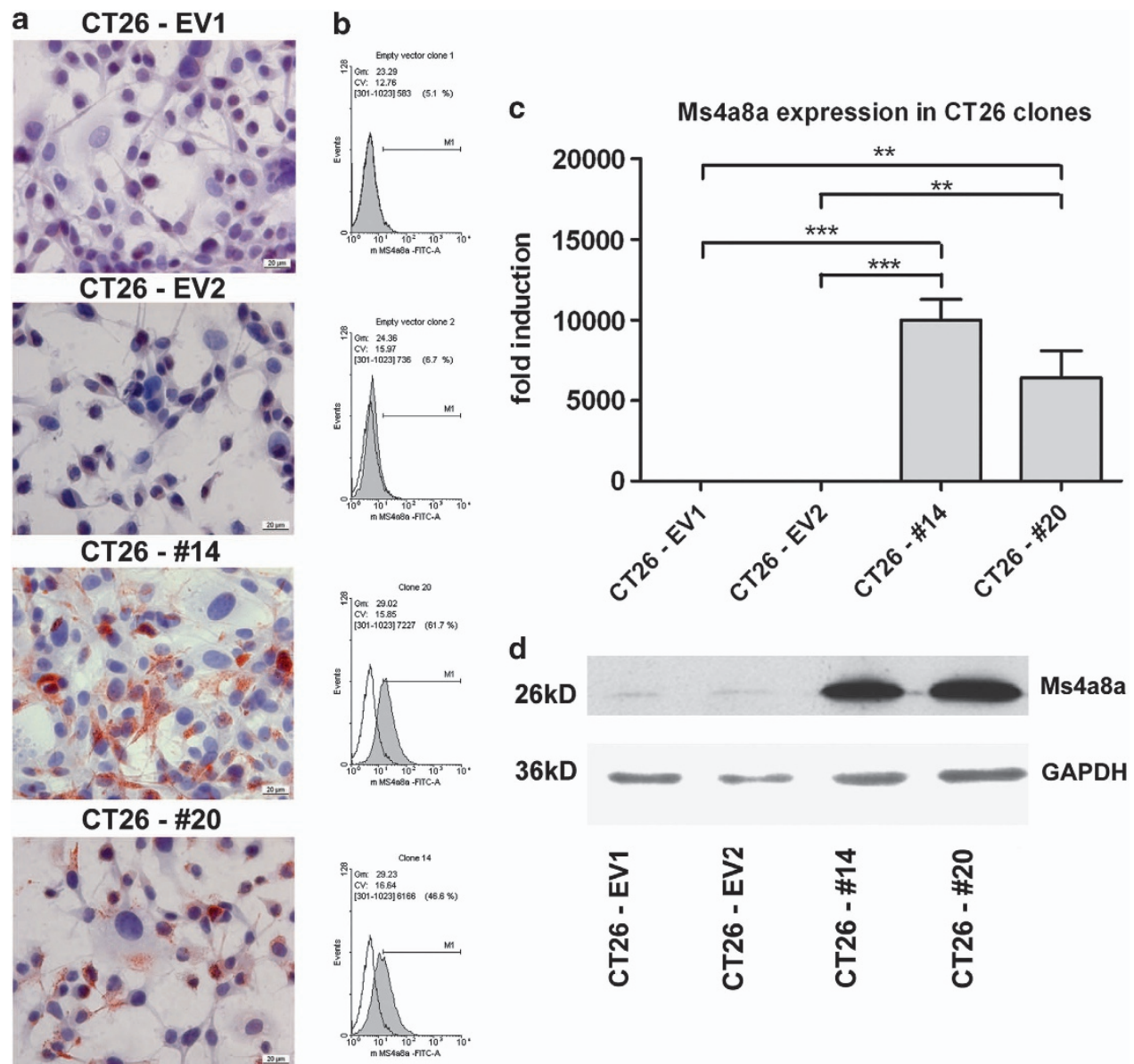

d
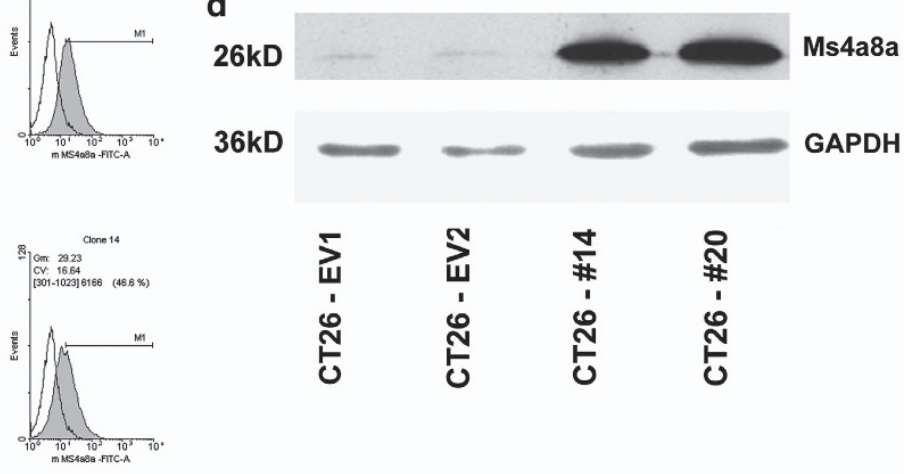

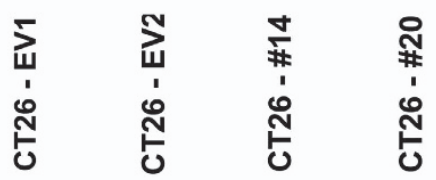

Figure 2 Verification of MS4A8A expression on protein and mRNA level in the transgenic murine colon carcinoma cell line CT26 transfected with either Ms4a8a vector DNA or empty vector DNA. (a) Histochemical stainings of acetone-fixed CT26 cells grown on cover slips with rabbit anti-mouse polyclonal MS4A8A antibody. Positive cells appear in red. Bar $=20 \mu \mathrm{m} ; n=3$. (b) FACS analysis of CT26-\#14, CT26-\#20, CT26-EV1 and CT26-EV2 with rabbit anti-mouse polyclonal MS4A8A antibody; $n=3$. (c) mRNA expression analyzed by quantitative reverse transcriptase (qRT)-PCR of CT26-\#14, CT26-\#20, CT26-EV1 and CT26-EV2. Values from qRT-PCR are given as means and S.E.M. for the expression of Ms4a8a relative to the housekeeping gene $\beta$-actin and are given as fold induction over CT26-EV1 set at $1 ; n=3$. (d) Western blot analysis of protein extracts of CT26-\#14, CT26-\#20, CT26-EV1 and CT26-EV2 with rabbit anti-mouse polyclonal MS4A8A antibody. For loading control samples were additionally stained with rabbit anti-mouse/human GAPDH antibody; $n=3$

organization, apoptosis, proliferation, differentiation and metabolic processes were found (Table 1). Beside krt20 only aldo-keto reductase family 1 member $\mathrm{C} 18$ showed a remarkable upregulation with a fold change $>10$. To verify the reliability of these results on protein and mRNA level, the differential expression levels of krt20 in CT26-\#14, CT26-\#20, CT26-EV1 and CT26- EV2 colon carcinoma cells were confirmed by quantitative reverse transcriptase-PCR (Figure 5a) and western blot (Figure $5 b$ ).

To compare the expression of KRT20, a well-known differentiation marker in the colon, with the one found for MS4A8A, healthy murine colon was stained. Interestingly, MS4A8A and KRT20 showed a similar expression profile in the upper part of the crypt-luminal axis, suggesting a codependency in the gut (Figures $5 \mathrm{c}$ and d).

\section{Discussion}

In our previous work, MS4A8A was found to be expressed by typical M2 macrophages in murine tumor tissue and parasitic infections. Forced overexpression of MS4A8A in a macrophage-like cell line led to the induction of a subset of genes involved in immune regulation but had no effect on proliferation or cell death. ${ }^{19,23}$

In this study, we show that not only MS4A8A is not restricted to macrophages but that it is also found in epithelial cells of the gastrointestinal tract. Thereby, MS4A8A as well as the human homolog MS4A8B were not detected in the stem cell niche of the gut but in differentiating cells. In addition, the expression of MS4A8B was lost in dedifferentiated human adenocarcinoma. These findings classify MS4A8A/MS4A8B as a novel marker for differentiation in the gut.

In the intestine, differentiation processes are tightly regulated by the combinational effect of positive and negative transcriptional regulatory networks and transcription factors such as HNF $4 \alpha$, CDX2, HNF $1 \alpha$ and many others. ${ }^{24}$ To identify known transcription factor-binding sites in the predicted Ms4a8a promoter, a bioinformatic analysis using Matinspector software was performed, which revealed two CDX2, one HNF $1 \alpha$, but no HNF4 $\alpha$-binding sites (data not shown). In the 
a

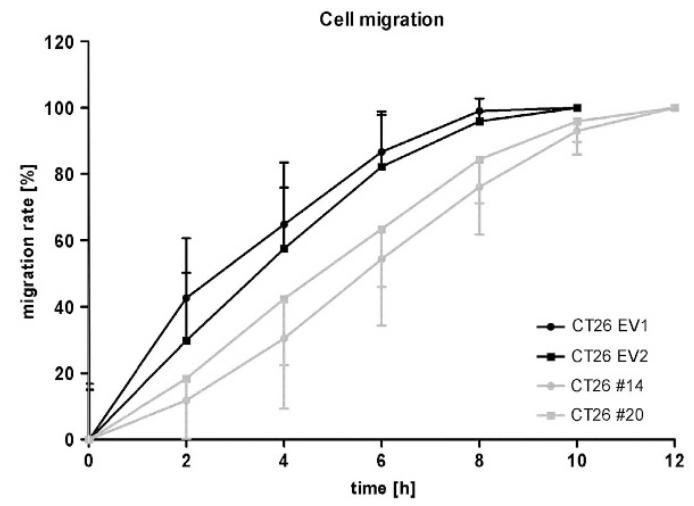

$0 \mathrm{~h}$
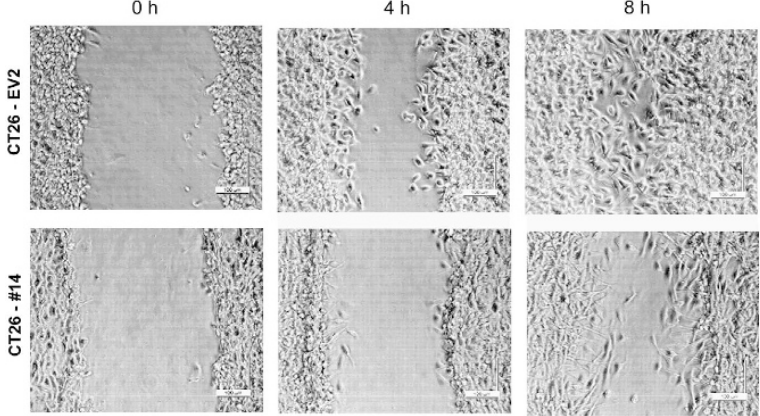

b

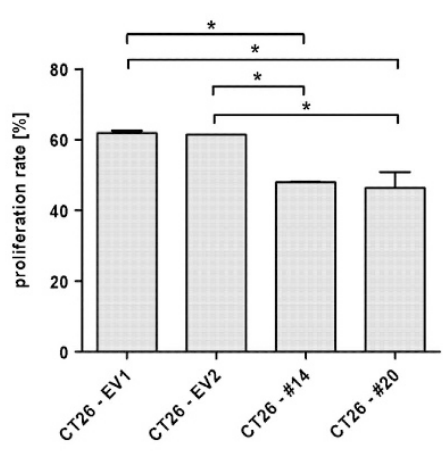

C

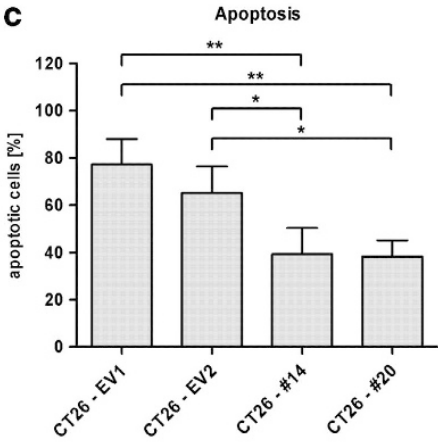

Figure 3 MS4A8A overexpression in CT26 cells has a negative impact on the ability of the cells to migrate and proliferate but increase apoptosis resistance. (a) Migration rates of mitomycin treated CT26-\#14, CT26-\#20, CT26-EV1 and CT26-EV2 were assessed by performing a scratch assay. Results are shown as percentages of three independent experiments, with $100 \%$ indicating a complete scratch closure. Representative pictures of three independent experiments are shown. Bar $=100 \mu \mathrm{m}$. (b) Proliferation rates of CT26-\#14, CT26-\#20, CT26-EV1 and CT26-EV2 were assessed by measuring the BrdU incorporation using fluorescence-activated cell sorter analysis. Results are shown as percentages of BrdU-positive CT26 cells. $n=3$. (c) Percentage of apoptotic CT26-\#14, CT26-\#20, CT26-EV1 and CT26-EV2 cells after a 3-h treatment with $2.5 \mathrm{mM} \mathrm{H}_{2} \mathrm{O}_{2} ; n=3$

gut, CDX2 and HNF1 $\alpha$ expression increases along the cryptsurface axis thereby inducing a rapid progression from proliferation to cell-cycle arrest. ${ }^{11}$ As overexpression of MS4A8A in CT26 cells leads to a statistically significant inhibition of proliferation and shows a similar expression pattern as CDX2 and $\mathrm{HNF} 1 \alpha$, a possible transcriptional regulation of $M S 4 A 8 A / M S 4 A 8 B$ by $C D X 2$ or $H N F 1 \alpha$ is imaginable.

Involvement of MS4A proteins in cell proliferation has already been described for CD20 (MS4A1) in B cells, MS4A3 in a human monocyte like cell line and for MS4A4B in T cells. ${ }^{25-29}$ Although CD20 seems to regulate cell-cycle progression by its regulatory function on transmembrane $\mathrm{Ca}^{2+}$ conductance, MS4A3 does this by binding to cyclindependent kinase-associated phospatase-cyclin-dependent kinase 2 complexes causing a G0/G1 cell-cycle arrest. ${ }^{28,30}$ For MS4A4B, the mechanism underlying the cell-cycle arrest in $T$ cells at the G0/G1 remains to be fully determined, but its overexpression correlates with an increase in cell-cycle inhibitors and a decrease in cyclins $A$ and $B .{ }^{29}$ How MS4A8A affects proliferation and migration in CT26 colon carcinoma cells still warrants clarification, as in contrast to many other MS4A proteins, MS4A8A lacks an intracytoplasmatic signaling sequence, which excludes direct pathway activation. The overall structure of MS4A8A, however, suggests a possible adaptor function as has been described for MS4A4B and MS4A6B. ${ }^{31}$ Using a yeast split-ubiquitin screen, Howie et al. $^{31}$ provided proof of a lateral association within the MS4A family members and with other co-stimulatory molecules as GITR (glucocorticoid-induced TNFR family-related gene). A cluster formation of MS4A4B with GITR was able to lower the threshold for GITR activation in regulatory T cells. In a similar manner, MS4A8A could interact with other surface receptors or other MS4A family members. In the gastrointestinal tract, several members of the MS4A family like Ms4a4, Ms4a6, Ms4a7, Ms4a10, Ms4a11 and Ms4a12 have been described. ${ }^{32}$ However, protein data are available only for MS4A12. ${ }^{20}$ On cellular level, MS4A8A/MS4A8B and MS4A12 both show a luminal distribution in a healthy colon, which makes a direct interaction possible.

MS4A8A overexpression in CT26 colon carcinoma cells induced a special gene signature comprising genes involved in cytoskeletal organization, apoptosis, proliferation, transcriptional regulation and, in particular, metabolic processes. This supports the hypothesis that MS4A8A might be involved in differentiation processes of intestinal epithelial cells as during differentiation along the crypt-surface axis upregulation of genes important for the cytoskeleton, digestive enzymes and transport proteins occur. ${ }^{9,33}$ The highest regulated gene by MS4A8A was Krt20, which was confirmed on the mRNA and protein level and showed an expression that parallels that of MS4A8A in the colon in vivo. KRT20 is a type I keratin and a well-known intestinal differentiation marker. ${ }^{7}$ It belongs to the keratin family, which provides important 
a

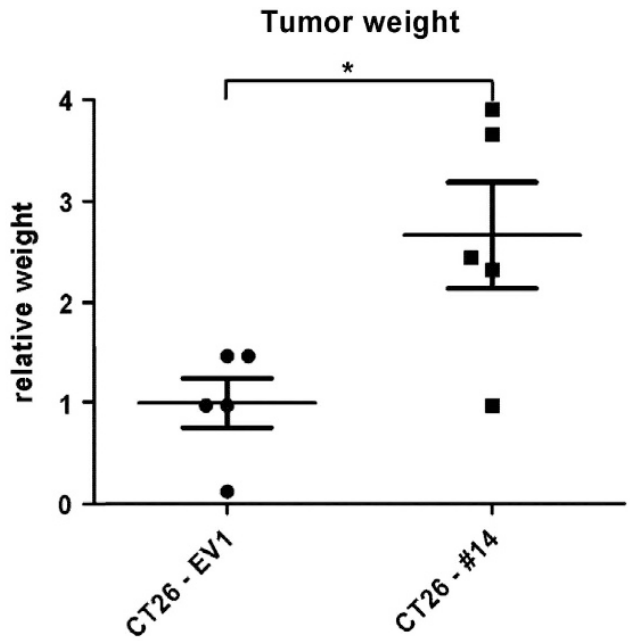

b

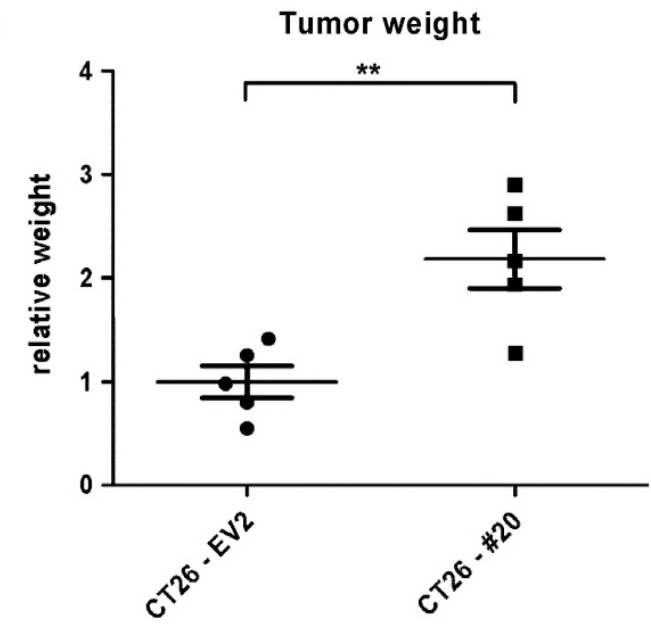

C

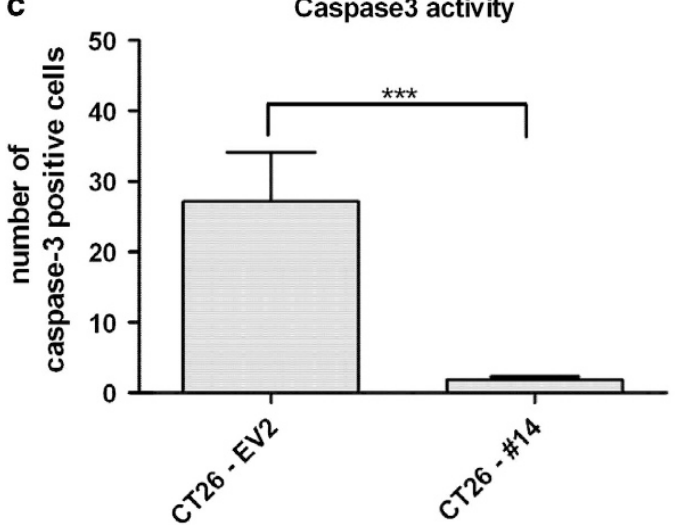

Figure 4 MS4A8A overexpression in CT26 cells leads to a higher tumor end weight in vivo by conferring increased resistance to apoptosis. (a) Relative end weights of 14 days old subcutaneously injected CT26-\#14 or CT26-EV1 tumors (b). Relative end weights of 21 days old subcutaneously injected CT26-\#20 or CT26EV2 tumors. The relative weight ( $\mathbf{a}$ and $\mathbf{b}$ ) is calculated by setting the end weight of CT26-EV1/2 as $1 ; n=5$. (c) Number of caspase 3-positive cells in subcutaneously injected CT26-\#14 and CT26-EV2 tumors ascertained by counting positive-stained cells of five randomly choosen $\times 40$ high power fields of each tumor; $n=3$

mechanical support for cells and protects them from apoptosis and non-mechanical forms of injury by interacting with different adaptor molecules and cell receptors. ${ }^{34}$
MS4A8A-transfected CT26 cells display an increased resistance to $\mathrm{H}_{2} \mathrm{O}_{2}$-induced apoptosis. Whether this effect and the effect on proliferation and migration are attributable directly to MS4A8A or rather to its regulated genes in particular KRT20 needs further evaluation.

Overall, the expression of MS4A8A during differentiation of intestinal epithelial cells along the crypt-luminal axis seems to support the integrity of the intestinal barrier function by modulating the transcriptome and conferring the intestinal cells an increased resistance to reactive oxygen species present in the gastrointestinal lumen. As we have shown that MS4A8B in humans is lost in colon carcinomas, MS4A8B in the future might also be used as marker to distinguish neoplastic from healthy tissue, but this needs further evaluation of tissue samples from colon carcinoma patients. Findings presented in this study might therefore help to further understand physiological and pathophysiological processes in the murine and human intestinal tract.

\section{Materials and Methods}

Mice. BALB/c wild type mice were purchased from Elevisier Janvier. Animal experimental protocols were approved by the animal ethics committee (Regierungspräsidium Karlsruhe, Az: 35-9185.81/G-115/07).

Human specimen. The use of human tissue for study purposes was approved by the local Ethics Committee at the University Hospital Heidelberg, Germany. Gut tissue samples were derived from surgical specimens of patients with established adenocarcinoma of the colon (Department of Surgery, University Hospital Heidelberg; informed consent was given by the patients). No patient received neoadjuvant therapy before operation. Diagnosis was made in accordance with clinical and conventional histopathological criteria. Tissue samples were obtained from macroscopically tumorous as well as macroscopically noninvolved gut regions and snap-frozen in liquid nitrogen. Subsequently, all samples included in the study underwent expert histopathological examination (LF) before further analysis. Clinical findings of the patients are provided in Table 2.

Materials/antibodies. The following reagents were used: rabbit polyclonal IgG anti GAPDH (glyceraldehyde-3-phosphate dehydrogenase; Santa Cruz Biotechnology, Santa Cruz, CA, USA), rabbit anti-mouse caspase 3 (Cell Signal Technology, Boston, MA, USA), monoclonal mouse anti-human/mouse Krt20 (clone Ks20.8; Dako, Glostrup, Denmark), guinea pig anti-mouse Krt20, goat antirabbit IgG-horseradish peroxidase (Santa Cruz Biotechnology), mouse BD Fc Block (BD Biosciences, Heidelberg, Germany), anti-mouse BrdU FITC and mouse IgG Isotype FITC (Sigma-Aldrich, Taufkirchen, Germany).

Custom-made anti-mouse MS4A8A serum was produced as previously described. ${ }^{19}$ Custom-made anti-MS4A8B serum was produced by immunizing rabbits with a synthetic peptide (234PVTSPPSYSSEIQANK250) representing the C-terminal part of MS4A8B (Peptide Speciality Laboratories, Heidelberg, Germany). Antibody specificity was confirmed by immunohistochemistry and western blot analysis of transient MS4A8B-transfected Caco2 cells and human gut.

Cells. CT26 colon carcinoma cells (ATCC CRL-2638) and Caco2 cells (ATCC HTB-37) were obtained from American Type Culture Collection. CT26 were cultivated with RPMI media with $10 \%$ FCS and $100 \mathrm{IU}$ penicillin, $100 \mu \mathrm{g} / \mathrm{ml}$ streptomycin. Caco2 were cultivated with DMEM media with $10 \%$ FCS and $100 \mathrm{IU}$ penicillin, $100 \mu \mathrm{g} / \mathrm{ml}$ streptomycin (all cell culture media Biochrom, Berlin, Germany). Authentication of cell lines was assured by regular morphology checks and growth curve analyses. Cells were regularly tested for mycoplasma infection by PCR.

Generation of Ms4a8a-transfected CT26 cells. A recombinant Ms4a8a cDNA was amplified by PCR (primer: Ms4a8a-Spel-fw 5'ATC GAATTCACTAGTAGCAAAGAGTTGGGAACCGGAGCAAGA3' and Ms4a8aNotl-rv: 5'ATATGCGGCCGCTAGAGCATCTTTAT3') from Ms4a8a cDNA RZPDp981B0530D (IMAGE ID 905005), purified on agarose gel and subcloned 
Table 1 Gene profiling of MS4A8A expressing CT26 colon carcinoma cells

\begin{tabular}{|c|c|c|c|c|}
\hline Gene Symbol & GeneName & UniGene HsID & Fold change $^{a}$ & Molecular function (UniProt) \\
\hline \multicolumn{5}{|c|}{ Genes upregulated by MS4A8A } \\
\hline Krt20 & Keratin 20 & Mm.28042 & 22,4 & Apotosis, cytoskeleton \\
\hline Akr1c18 & Aldo-keto reductase family 1 , member $\mathrm{C} 18$ & Mm.41337 & 12,7 & Metabolic processes \\
\hline AU018778 & Expressed sequence AU018778 & Mm.29110 & 9,5 & Metabolic processes \\
\hline Prkd1 & Protein kinase D1 & Mm.133719 & 6,1 & Apotosis, differentiation \\
\hline Akr1c14 & Aldo-keto reductase family 1 , member $\mathrm{C} 14$ & Mm.26838 & 5,4 & Differentiation \\
\hline C1s & complement component 1 , s subcomponent & Mm.219527 & 5,3 & Immune response \\
\hline Psmb8 & Proteasome subunit, beta type 8 & Mm.180191 & 4,7 & Differentiation \\
\hline Slco1a5 & Solute carrier organic anion transporter family, member 1 a5 & Mm.206599 & 4,3 & Metabolic processes \\
\hline Ms4a8a & Membrane-spanning 4-domains, subfamily A & Mm.34460 & 4,1 & Receptor activity \\
\hline Btc & Betacellulin, epidermal growth factor family member & Mm.2024 & 3,9 & Proliferation, apoptosis \\
\hline Bmper & BMP-binding endothelial regulator & Mm.335020 & 3,9 & Angiogenesis \\
\hline Cask & Calcium/calmodulin-dependent serine protein kinase & Mm.327591 & 3,8 & Metabolic processes \\
\hline Ccl9 & Chemokine (C-C motif) ligand 9 & Mm.416125 & 3,7 & Immune response \\
\hline Mapk12 & Mitogen-activated protein kinase 12 & Mm.38343 & 3,7 & Transcriptional regulation \\
\hline C1ra & Complement component $1, \mathrm{r}$ subcomponent $\mathrm{A}$ & Mm.333375 & 3,7 & Immune response \\
\hline Ceacam1 & Carcinoembryonic antigen-related cell adhesion molecule 1 & Mm.322502 & 3,4 & Immune response \\
\hline Ndrg1 & $\mathrm{N}$-myc downstream regulated gene 1 & Mm.30837 & 3,4 & DNA repair \\
\hline Thbs2 & Thrombospondin 2 & $\mathrm{Mm} .26688$ & 3,3 & Cytoskeletal organization \\
\hline Rp2h & Retinitis pigmentosa 2 homolog (human) & Mm.288141 & 3,3 & Transcriptional regulation \\
\hline Ebp & Phenylalkylamine $\mathrm{Ca} 2+$ antagonist-binding protein & Mm.27183 & 3,3 & Metabolic processes \\
\hline Atp6ap2 & ATPase, $\mathrm{H}+$ transporting, lysosomal accessory protein 2 & Mm.25148 & 3,2 & Differentiation \\
\hline Plscr2 & Phospholipid scramblase 2 & Mm.10306 & 3,2 & Metabolic processes, apoptosis \\
\hline Dynlt3 & Dynein light chain Tctex-type 3 & Mm.29150 & 3,2 & Proliferation \\
\hline Fam84b & Family with sequence similarity 84 , member B & Mm.230853 & 3,1 & Protein binding \\
\hline Arhgap6 & Rho GTPase-activating protein 6 & Mm.441810 & 3,1 & Cytoskeletal organization \\
\hline Rarb & Retinoic acid receptor, beta & Mm.259318 & 3,0 & Transcriptional regulation \\
\hline Ifi204 & Interferon-activated gene 204 & Mm.261270 & 3,0 & Transcriptional regulation \\
\hline \multicolumn{5}{|c|}{ Genes downregulated by MS4A8A } \\
\hline Pkdcc & Protein kinase domain containing, cytoplasmic & Mm.311974 & 0,4 & Differentiation \\
\hline Mum1l1 & Melanoma-associated antigen (mutated) 1-like 1 & Mm.131001 & 0,4 & Not known \\
\hline Zdhhc14 & Zinc finger, DHHC domain containing 14 & Mm.311028 & 0,4 & Metabolic processes \\
\hline Ddah1 & Dimethylarginine dimethylaminohydrolase 1 & Mm.234247 & 0,4 & Metabolic processes \\
\hline Rps6ka1 & Ribosomal protein S6 kinase polypeptide 1 & Mm.301827 & 0,4 & Metabolic processes \\
\hline Bnc2 & Basonuclin 2 & Mm.190774 & 0,3 & Transcriptional regulation \\
\hline Nap112 & Nucleosome assembly protein 1-like 2 & Mm.439770 & 0,3 & Not known \\
\hline Adam12 & A disintegrin and metallopeptidase domain 12 & Mm.439714 & 0,3 & Adhesion \\
\hline A230050P20 Rik & RIKEN cDNA A230050P20 gene & Mm.249932 & 0,2 & Not known \\
\hline
\end{tabular}

aold induction of genes differentially expressed by MS4A8A + CT26-\#14 and CT26-\#20 versus Ms4a8a- CT26-EV1 and CT26-EV2 ( $\mathrm{n}=3$ ). Only genes with a fold change of $\geq 3$ for upregulation and $\leq 0.5$ for downregulation are presented.

after digestion with Spel and Notl restriction enzymes into the expression vector pEF6/V5-His Topo (Invitrogen, Carlsbad, CA, USA) according to standard molecular biology protocols. After confirming sequence identity, we transfected CT26 colon carcinoma cells with Ms4a8a vector DNA using Lipofectamine 2000 (Invitrogen) transfection reagent. Transfectants were selected by resistance to blasticidin (Invitrogen). CT26-\#14 and CT26-\#20 clones with recombinant Ms4a4a expression were propagated. As a negative control, two vector-transfected CT26 clones (CT26-EV1 and CT26-EV2) were selected under parallel culture conditions.

A recombinant Ms4a8b cDNA was amplified by PCR (primer: Ms4a8b-Spel-fw 5'GATCACTAGTACATTCATTCTC3' and Ms4a8b-Notl-rv: 5'GATCGCGGCCGC TGTCAGAACAGGAA3') from Ms4a8b cDNA IRAUp969G0588D (IMAGE ID 4691480) purified on agarose gel and subcloned after digestion with Spel and Notl restriction enzymes into the expression vector pEF6/V5-His Topo (Invitrogen) according to standard molecular biology protocols. After confirming sequence identity, Caco2 cells were transiently transfected with Ms4a8b vector DNA using ExtremeGene9 (Roche Diagnostics, Mannheim, Germany) transfection reagent.

Tumor models. A total of $3 \times 10^{5}$ transgenic CT26 cells (CT26-EV1, CT26EV2, CT26-\#14 and CT26-\#20) were injected subcutaneously into the right flank of BALB/c mice. After 14 or 21 days, animals were killed. Tumors were harvested weighted and afterwards snap-frozen in liquid nitrogen. Animal experimental protocols were approved by the animal ethics committee (Regierungspräsidium Karlsruhe, Az: 35-9185.81/G-208/10).
Immunohistochemistry. Cryostat sections of murine and human colon and human colon adenocarcinoma as well as cells grown on glass cover slips were air-dried and acetone-fixed, blocked with $2 \%$ BSA in PBS and incubated with the first antibody. The appropriate horseradish peroxidase-labeled secondary antibody was used. Pictures were taken with a Leica DCRE microscope, Leica DC500 camera and software system (Leica, Wetzlar, Germany). Images were arranged using Photoshop 6.0 software (Adobe, San Jose, CA, USA)

Western blot analysis. Proteins were obtained by treating whole cells or tissue with RIPA-P buffer ( $150 \mathrm{mM} \mathrm{NaCl}, 15 \mathrm{NP} 40,0,5 \%$ sodium desoxycholate, $0,1 \%$ SDS, $50 \mathrm{mM}$ Tris/HCL (pH 8.0), $10 \mathrm{mM} \mathrm{NaF}, 1 \mathrm{mM} \mathrm{Na}_{3} \mathrm{OV}_{4}, 2 \mathrm{mM}$ EDTA (all Sigma-Aldrich)) enriched with a protease inhibitor cocktail (Roche Diagnostics). Equal amounts of protein were carried on a $12 \%$ SDS polyacrylamide gel. After blotting onto nitrocellulose (Schleicher\&Schüll Bioscence, Dassel, Germany), membranes were incubated for $3 \mathrm{~h}$ with the MS4A8A, MS4A8B, KRT20 or GAPDH antibody respectively, followed by incubation with the appropriate secondary horseradish peroxidase-labeled antibody. For signal detection, SuperSignal West Pico Chemiluminescent Substrate (Pierce Biotechnology, Rockford, IL, USA) was used.

Quantitative reverse transcriptase-PCR analysis. For RNA extraction, the Total RNA Kit I (Omni Life Science GmbH, Hamburg, Germany) was used according to the manufacturer's instructions. For cDNA synthesis, $1 \mu \mathrm{g}$ 
a

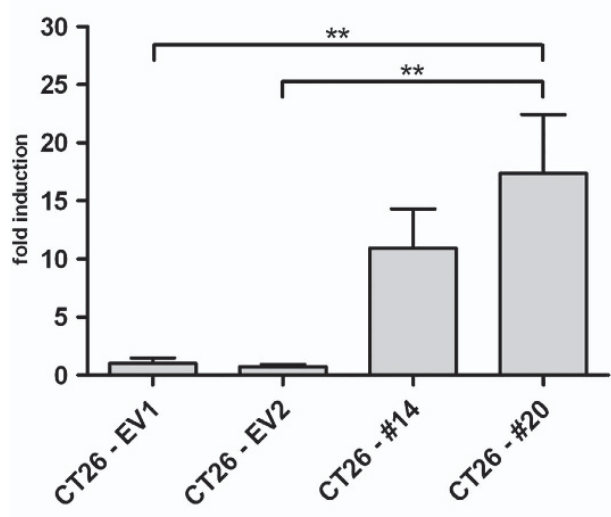

b

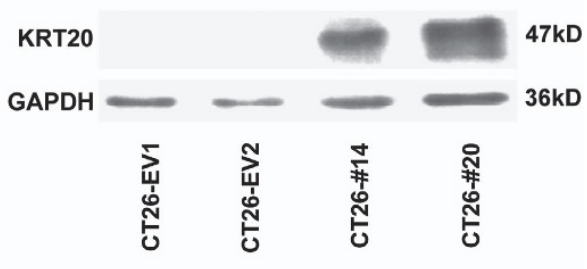

d murine large intestine
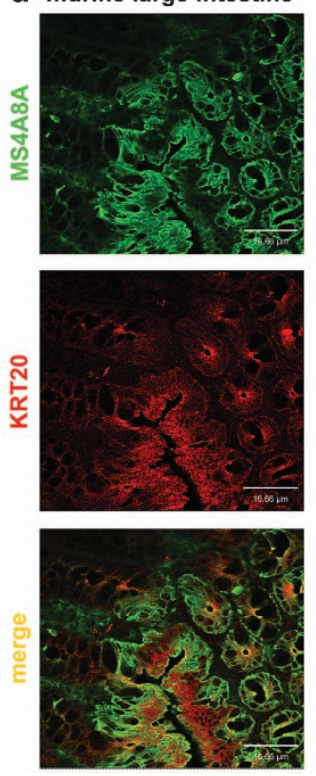

Figure 5 MS4A8A induces the expression of KRT20 in CT26 cells on mRNA and protein level. In addition, an overlapping expression of MS4A8A and KRT20 is found in vivo in the murine large intestine. (a) mRNA expression of Krt20 analyzed by quantitative reverse transcriptase (qRT)-PCR of CT26-\#14, CT26-\#20, CT26-EV1 and CT26EV2. Values from qRT-PCR are given as means and S.E.M. for the expression of Ms4a8a relative to the housekeeping gene $\beta$-actin and are given as fold induction over CT26EV1 set at 1; $n=3$. (b) Western blot analysis of protein extracts from CT26-\#14, CT26-\#20, CT26-EV1 and CT26-EV2 stained with guinea pig anti-human/mouse KRT20 antibody. To ensure identical protein loading, the same samples were stained with rabbit anti-mouse/human GAPDH antibody; $n=3$. (c) Histochemical stainings of acetonefixed cryostat sections of murine large intestine with rabbit anti-mouse polyclonal MS4A8A and mouse anti-human/mouse KRT20 antibody as indicated. Positive cells appear in red. Bars $=100 \mu \mathrm{m}$ and $50 \mu \mathrm{m} . n=3$. (d) Immunofluorescent double staining of the murine large intestine with rabbit anti-mouse MS4A8A (green) and mouse anti-human/ mouse KRT20 antibody (red). Images were acquired using confocal microscopy. Bars $=16.66 \mu \mathrm{m} ; n=3$

Table 2 Background information on human adenocarcinomas

\begin{tabular}{lcccc}
\hline & & \multicolumn{2}{c}{ Human tissue samples } \\
\hline Tissue & Gender & Age & Diagnosis & TNM \\
\hline Cecum & $M$ & 67 & Adenocarcinoma & pT2, pN0(0/13), pMx, L0, V0 \\
Cecum & $M$ & Adenocarcinoma & pT1, pN0(0/28), pMx, L0, V0 \\
Colon descendens & $M$ & 59 & Adenocarcinoma & GT3, pN0 (0/12), pMx, L0, V0 \\
\hline
\end{tabular}

RNA was used for reverse transcription with RevertAid H Minus M-MuLV Reverse Transcriptase (Fermentas, St Leon-Rot, Germany) using Oligo (dT) ${ }_{18}$ Primer following the manufacturer's instructions.

For quantitative reverse transcriptase-PCR, $1 \mu \mathrm{l}$ of CDNA was amplified using SyBRGreen PCR Master Mix (Applied Biosystems Inc., Carlsbad, CA, USA) under standard conditions with an MX3000P sequence detection system (Stratagene, La Jolla, CA, USA). All primers used are listed in Supplementary Table S1.
FACS analysis. For FACS analysis, $5 \times 10^{6}$ cells were washed three times with PBS one time and fixed with $4 \%$ paraformaldehyde. First antibody (MS4A8A or MS4A8B) was incubated for $1 \mathrm{~h}$ on ice. After washing with PBS, the secondary antibody (goat anti-rabbit FITC) was added. Fluorescence labeled cells were analyzed with FACS-Cantoll (BD Biosciences, Heidelberg, Germany). Results were analyzed with the WinMDI Version 2.8 software (www.winmdi.software.informer.com). 
Proliferation assay. In all, $1 \times 10^{6}$ cells were seeded in a six-well plate, allowed to grow until $70-80 \%$ confluency. To measure DNA synthesis as indicator for cell division $25 \mu \mathrm{g} / \mathrm{ml} \mathrm{BrdU} \mathrm{(from} \mathrm{Sigma-Aldrich)} \mathrm{was} \mathrm{added} \mathrm{to} \mathrm{the} \mathrm{cell} \mathrm{culture}$ for $1 \mathrm{~h}$. After washing, free BrdU cells were trypsinated, fixed with $70 \%$ ethanol and denatured with $2 \mathrm{M} \mathrm{HCl}$. After denaturation, the cells were neutralized with $0.1 \mathrm{M}$ sodiumborat buffer and incubated with FITC-labeled mouse anti-BrdU or a mouse IgG isotype control. Fluorescence was detected by flowcytometry (FACS Canto II) and afterwards analyzed by WinMDI Version 2.8 software.

Apoptosis assay with crystal violet. In all, $7 \times 10^{4}$ cells were seeded in a 96-well plate (covered with $0.2 \%$ gelatin in $\mathrm{H}_{2} \mathrm{O}$ ) and incubated until complete cell confluence was reached. Subsequently, cells were incubated for $3 \mathrm{~h}$ with $2.5 \mathrm{mM} \mathrm{H}_{2} \mathrm{O}_{2}$ or control media, stained and fixed for 15 min with $5 \%$ crystal violet in $20 \%$ methanol and air-dried. Crystal violet-stained cells were dissolved in $100 \%$ methanol and photometrically evaluated with the multilabel counter Wallac 1420 (PerkinElmer, Waltham, MA, USA).

Scratch migration assay. In all, $2.5 \times 10^{4}$ cells were grown to $100 \%$ confluence on a 12-well plate coated with $0.2 \%$ gelatin. After a pre-treatment with $10 \mathrm{ng} / \mathrm{ml}$ mitomycin C (Carl Roth GmbH, Karlsruhe, Germany) for 45 min to inhibit proliferation, a scratch was set with a pipette tip in the cell monolayer, and the floating cells were washed away by several changes of media. Scratches were photographed every $2 \mathrm{~h}$ until complete closure. For each time point, five pictures of each scratch were randomly taken. In each picture, five measuring bars were set using Image $\mathrm{J}$ software (freeware, http://rsbweb.nih.gov/ij/). Results are shown as percentages with $100 \%$ defined as a complete wound closure.

Caspase activity. A representative cryostat section of three CT26-\#14 and three CT26-\#EV2 tumors was stained as previously described with anti-mouse caspase 3 antibody and the appropriate horseradish peroxidase-labeled secondary antibody. Five pictures of each tumor were randomly taken and the number of caspase 3-positive cells was counted. Necrotic areas were excluded from the cell count.

cDNA microarray analysis and statistical procedures. For microarray analysis, $10 \times 10^{6} \mathrm{Ms} 4 a 8 \mathrm{a}$-transfected and EV-transfected CT26 cells were incubated with RPMI, $10 \% \mathrm{FCS}, 100 \mathrm{IU}$ penicillin, $100 \mu \mathrm{g} / \mathrm{ml}$ streptomycin for $48 \mathrm{~h}$. Gene expression profiling was performed using mouse genome 4302.0 DNA arrays (Affymetrix, Santa Clara, CA, USA) according to the recommendations of the manufacturer. A Custom CDF Version 14 with Entrez-based gene definitions was used to annotate the arrays. Differential gene expression was analyzed based on loglinear mixed model ANOVA using a commercial software package SAS JMP7 Genomics, version 4 from SAS (SAS Institute, Heidelberg, Germany). A false-positive rate of $a=0.05$ with FDR correction was taken as the level of significance. Full data are deposited in the Gene Expression Omnibus database.

Statistical analysis. All data were statistically evaluated by using GraphPad Prism 5.0 (GraphPad Software, San Diego, CA, USA). Statistical Significance was verified by using one-way ANOVA and Bonferroni as post test. For the scratch migration assay, area under the curve was calculated and statistically evaluated using one-way ANOVA and Bonferroni post test. Level of significance was indicated by asterisks ${ }^{* * *}<0.001 ;{ }^{* *}<0.01$; and $\left.{ }^{*}<0.05\right)$. Error bars show S.E.M. of each experiment. All experiments were performed at least in triplicates.

\section{Conflict of Interest}

All authors declare no conflict of interest.

Acknowledgements. This work was supported, in part, by grants of Deutsche Forschungsgemeinschaft SFB938, project $\mathrm{H}$ to $S G$ and project $Z 2$ to FL. We thank Christina Schmuttermaier and Evelyn Exner for expert technical assistance.

1. Sandle Gl. Salt and water absorption in the human colon: a modern appraisal. Gut 1998; 43: 294-299

2. Papetti M, Augenlicht LH. MYBL2, a link between proliferation and differentiation in maturing colon epithelial cells. J Cell Physiol 2010; 226: 785-791.
3. Shie JL, Chen ZY, O'Brien MJ, Pestell RG, Lee ME, Tseng CC. Role of gut-enriched Kruppel-like factor in colonic cell growth and differentiation. Am J Physiol Gastrointest Liver Physiol 2000; 279: G806-G814.

4. Katz JP, Perreault N, Goldstein BG, Lee CS, Labosky PA, Yang VW et al. The zinc-finger transcription factor Klf4 is required for terminal differentiation of goblet cells in the colon. Development 2002; 129: 2619-2628.

5. Lueck A, Brown D, Kwiatkowski DJ. The actin-binding proteins adseverin and gelsolin are both highly expressed but differentially localized in kidney and intestine. J Cell Sci 1998; 111(Pt 24): 3633-3643.

6. Colnot S, Romagnolo B, Lambert M, Cluzeaud F, Porteu A, Vandewalle A et al. Intestinal expression of the calbindin-D9K gene in transgenic mice. Requirement for a Cdx2-binding site in a distal activator region. J Biol Chem 1998; 273: 31939-31946.

7. Chan CW, Wong NA, Liu Y, Bicknell D, Turley H, Hollins L et al. Gastrointestinal differentiation marker Cytokeratin 20 is regulated by homeobox gene CDX1. Proc Natl Acad Sci USA 2009; 106: 1936-1941.

8. Lonnerholm G, Selking O, Wistrand PJ. Amount and distribution of carbonic anhydrases CA I and CA II in the gastrointestinal tract. Gastroenterology 1985; 88 (5 Pt 1): 1151-1161.

9. Mariadason JM, Nicholas C, L'Italien KE, Zhuang M, Smartt HJ, Heerdt BG et al. Gene expression profiling of intestinal epithelial cell maturation along the crypt-villus axis. Gastroenterology 2005; 128: 1081-1088.

10. Hall PA, Coates PJ, Ansari B, Hopwood D. Regulation of cell number in the mammalian gastrointestinal tract: the importance of apoptosis. J Cell Sci 1994; 107(Pt 12): 3569-3577.

11. Olsen AK, Boyd M, Danielsen ET, Troelsen JT. Current and emerging approaches to define intestinal epithelium-specific transcriptional networks. Am J Physiol Gastrointest Liver Physiol 2012; 302: G277-G286.

12. Jensen J, Pedersen EE, Galante $P$, Hald J, Heller RS, Ishibashi $M$ et al. Control of endodermal endocrine development by Hes-1. Nat Genet 2000; 24: 36-44.

13. Batlle E, Henderson JT, Beghtel H, van den Born MM, Sancho E, Huls G et al. Beta-catenin and TCF mediate cell positioning in the intestinal epithelium by controlling the expression of EphB/ephrinB. Cell 2002; 111: 251-263.

14. Kinzler KW, Vogelstein B. Lessons from hereditary colorectal cancer. Cell 1996; 87: 159-170.

15. Pinto D, Clevers H. Wnt, stem cells and cancer in the intestine. Biol Cell 2005; 97: 185-196.

16. Barros R, Pereira B, Duluc I, Azevedo M, Mendes N, Camilo V et al. Key elements of the BMP/SMAD pathway co-localize with CDX2 in intestinal metaplasia and regulate CDX2 expression in human gastric cell lines. J Pathol 2008; 215: 411-420.

17. Pino MS, Kikuchi H, Zeng M, Herraiz MT, Sperduti I, Berger D et al. Epithelial to mesenchymal transition is impaired in colon cancer cells with microsatellite instability. Gastroenterology 2010; 138: 1406-1417.

18. Alberici P, Fodde R. The role of the APC tumor suppressor in chromosomal instability. Genome Dyn 2006; 1: 149-170.

19. Schmieder A, Schledzewski K, Michel J, Tuckermann JP, Tome L, Sticht C et al. Synergistic activation by p38MAPK and glucocorticoid signaling mediates induction of M2like tumor-associated macrophages expressing the novel CD20 homolog MS4A8A. Int J Cancer 2011; 129: 122-132.

20. Koslowski M, Sahin U, Dhaene K, Huber C, Tureci O. MS4A12 is a colon-selective storeoperated calcium channel promoting malignant cell processes. Cancer Res 2008; 68: 3458-3466.

21. Kutok JL, Yang X, Folkerth R, Adra CN. Characterization of the expression of HTm4 (MS4A3), a cell cycle regulator, in human peripheral blood cells and normal and malignant tissues. J Cell Mol Med 2011; 15: 86-93.

22. Liang Y, Tedder TF. Identification of a CD20-, FcepsilonRIbeta-, and HTm4-related gene family: sixteen new MS4A family members expressed in human and mouse. Genomics 2001; 72: 119-127.

23. Schmieder A, Schledzewski K, Michel J, Schonhaar K, Morias $\mathrm{Y}$, Bosschaerts $\mathrm{T}$ et al. The CD20 homolog Ms4a8a integrates pro- and anti-inflammatory signals in novel M2-like macrophages and is expressed in parasite infection. Eur J Immunol 2012; 42: 2971-2982.

24. Traber PG, Silberg DG. Intestine-specific gene transcription. Annu Rev Physiol 1996; 58: 275-297.

25. Kanzaki M, Shibata H, Mogami H, Kojima I. Expression of calcium-permeable cation channel CD20 accelerates progression through the G1 phase in Balb/c 3T3 cells. J Biol Chem 1995; 270: 13099-13104.

26. Tedder TF, Forsgren A, Boyd AW, Nadler LM, Schlossman SF. Antibodies reactive with the B1 molecule inhibit cell cycle progression but not activation of human B lymphocytes. Eur $J$ Immunol 1986; 16: 881-887.

27. Golay J, Cusmano G, Introna M. Independent regulation of c-myc, B-myb, and c-myb gene expression by inducers and inhibitors of proliferation in human B lymphocytes. $J$ Immuno 1992; 149: 300-308.

28. Donato JL, Ko J, Kutok JL, Cheng T, Shirakawa T, Mao XQ et al. Human HTm4 is a hematopoietic cell cycle regulator. J Clin Invest 2002; 109: 51-58.

29. Xu H, Yan Y, Williams MS, Carey GB, Yang J, Li H et al. MS4a4B, a CD20 homologue in T cells, inhibits T cell propagation by modulation of cell cycle. PLoS One 2011; 5 : e13780 
34. Coulombe PA, Wong P. Cytoplasmic intermediate filaments revealed as dynamic and multipurpose scaffolds. Nat Cell Biol 2004; 6: 699-706. molecule into ectopic cell types generates a $\mathrm{Ca} 2+$ conductance found constitutively in $\mathrm{B}$ lymphocytes. J Cell Biol 1993; 121: 1121-1132.

31. Howie D, Nolan KF, Daley S, Butterfield E, Adams E, Garcia-Rueda $\mathrm{H}$ et al. MS4A4B is a GITR-associated membrane adapter, expressed by regulatory $T$ cells, which modulates T cell activation. J Immunol 2009; 183: 4197-4204.

32. Ishibashi K, Suzuki M, Sasaki S, Imai M. Identification of a new multigene fourtransmembrane family (MS4A) related to $\mathrm{CD} 20, \mathrm{HTm} 4$ and beta subunit of the high-affinity IgE receptor. Gene 2001; 264: 87-93.

33. Halbleib JM, Saaf AM, Brown PO, Nelson WJ. Transcriptional modulation of genes encoding structural characteristics of differentiating enterocytes during development of a polarized epithelium in vitro. Mol Biol Cell 2007; 18: 4261-4278.
Cell Death and Disease is an open-access journal published by Nature Publishing Group. This work is licensed under the Creative Commons Attribution-NonCommercial-No Derivative Works 3.0 Unported License. To view a copy of this license, visit http://creativecommons.org/licenses/by-nc-nd/3.0/

Supplementary Information accompanies the paper on Cell Death and Disease website (http://www.nature.com/cddis) 